\title{
Extension of Fermat's last theorem in Minkowski natural spaces
}

\section{Ramon Carbó-Dorca ${ }^{1}\left[\right.$ · Sebastián Reyes ${ }^{2} \cdot$ Alfonso Niño $^{3}$}

Received: 24 May 2021 / Accepted: 21 June 2021

(c) The Author(s) 2021

\begin{abstract}
Minkowski natural $(N+1)$-dimensional spaces constitute the framework where the extension of Fermat's last theorem is discussed. Based on empirical experience obtained via computational results, some hints about the extension of Fermat's theorem from $(2+1)$-dimensional Minkowski spaces to $(N+1)$-dimensional ones. Previous experience permits to conjecture that the theorem can be extended in $(3+1)$ spaces, new results allow to do the same in $(4+1)$ spaces, with an anomaly present here but difficult to find in higher dimensions. In $(N+1)$ dimensions with $N>4$ there appears an increased difficulty to find Fermat vectors, there is discussed a possible source of such an obstacle, separately of the combinatorial explosion associated to the generation of natural vectors of high dimension.
\end{abstract}

Keywords $(N+1)$-dimensional natural Minkowski spaces $\cdot$ Extension of Fermat's last theorem · Fermat hypersurfaces · Conjectures on extended Fermat's theorem

Ramon Carbó-Dorca

ramoncarbodorca@gmail.com

Sebastián Reyes

sebastian.reyes@uclm.es

Alfonso Niño

Alfonso.nino@uclm.es

1 Institut de Química Computacional I Catàlisi, Universitat de Girona, Campus de Montilivi, 17003 Girona, Catalonia, Spain

2 Escuela Superior de Informatica, Universidad de Castilla-La Mancha, Paseo de La Universidad 4, 13004 Ciudad Real, Spain

3 Facultad de Ciencias Sociales, Universidad de Castilla-La Mancha, Talavera de La Reina, Avda. Real Fabrica de Sedas S/N, 45600 Toledo, Spain 


\section{Introduction}

Boolean hypercubic structure, Natural vector spaces, Minkowski spaces, and definition of generalized scalar products permitted to describe a large set of applications, which can be utilized to various chemical problems in general, but mainly associated with QSPR, see for example reference [1-3], for recently published papers on this subject. The present paper constitutes the theoretical part of a project encompassing a time-consuming computational effort and can be broadly located within the set of mathematical applications to chemistry and physics.

Three previous papers have been devoted to the problem of extending Fermat's last theorem. The initial one was almost purely theoretical [4] and was setting up the problem, the second paper was backed up with computational information [5] and constituted a new step into Fermat's theorem extension, and the third work recently published, presented an extended supercomputational framework to cope with the problem as far as possible [6].

Results of this last paper permitted to conjecture a behavior in three-dimensional spaces of natural vectors, similar to the property associated with vectors in two-dimensional natural spaces leading to Fermat's last theorem.

Fermat's theorem comportment in higher dimensional natural spaces was not discussed with the aid of the information gathered from various computational sources yet. The present study, using a sufficiently large set of varied dimensions, will discuss the number of Fermat vectors found and the conjectures that one can imagine about the extension of Fermat's last theorem to higher dimensions as well.

The existence of Fermat vectors in any dimension of natural spaces is connected with the $p$-th order norms of the natural vectors, see references $[4,5]$. The original Fermat's theorem might be seen as a property of natural two-dimensional vectors and the numerical behavior of their Euclidean and higher-order norms. In this case, if one wants to extend Fermat's theorem to any power and dimension, such endeavor can be explicitly described using generalized natural vector norms.

However, the norm property making some vector a Fermat one, that is: that the $p$-th order natural vector norm equals the $p$-th power of a natural number, which can be easily associated to $N$-dimensional Euclidean natural spaces, might be also connected to $(N+1)$-dimensional Minkowski natural spaces. Such spaces have been recently introduced in several papers, see for example references [4, 12, 15] and will be used systematically here.

Therefore, the theoretical body of this paper will be constructed by Minkowski spaces rather than Euclidean spaces. In this form, Fermat's last theorem can be conjectured for arbitrary dimensional Minkowski spaces. Thus, one can define Fermat's vectors compliant with Fermat's extended theorem as natural Minkowski vectors with zero $p$-th order norms.

The present work is assembled with the description of natural spaces and the useful operations to construct a sound structure to study the extension of Fermat's last theorem. Focus is made on the way to compute natural vector norms of any order because such mathematical operations constitute the background of the extended Fermat theorem. 
This preliminary description allows describing the Minkowski spaces of dimension $(N+1)$, essential to define Fermat vectors via norms with zero value. After this follows an analysis of the extended Fermat's theorem conjectures one can construct from empirical computational experience.

A final discussion about the increasing scarcity of Fermat's vectors when Minkowski space dimensions augment finishes this paper.

\section{Natural Spaces}

A natural $N$-dimensional space is a vector space defined on the natural number set $\mathbb{V}_{N}[\mathbb{N}]$. There might be axiomatized that the natural spaces possess an addition semigroup, lacking subtraction, and negative numbers, as the natural number set does. In this sense, natural spaces might be also called semispaces. As, in general, vector spaces attached to such an addition structure have been named in previous literature [7].

Also, natural vector spaces can be easily associated with two characteristics: (1) the inward product [7-10] operation and (2) the complete sum operator [11]. Such an operation and operator permit the easy definition of powers of a vector, generalized scalar products, and $p$-th order norms [12]. A resumé of both follows so that the readers can avoid perusing the literature on this subject, and thus the present study becomes self-contained.

\subsection{Inward product of two natural vectors}

By the inward product of two vectors is constructed another vector of the same space where the factor vectors belong. The resultant inward product vector elements are the product of the elements of the implied vectors. That is, using for row vectors a Dirac's bra notation, or:

$$
\forall\langle\mathbf{a}| \in \mathbb{V}_{N}[\mathbb{N}]:\langle\mathbf{a}|=\left\{a_{I} \mid I=1, N\right\}=\left(a_{1}, a_{2}, a_{3}, \ldots a_{N}\right),
$$

then an inward product $\langle\mathbf{p}|=\langle\mathbf{a}| *\langle\mathbf{b}|$ between two vectors is defined as:

$$
\begin{aligned}
& \forall\left\{\langle\mathbf{a}|=\left\{a_{I} \mid I=1, N\right\},\langle\mathbf{b}|=\left\{b_{I} \mid I=1, N\right\}\right\} \subset \mathbb{V}_{N}[\mathbb{N}] \Rightarrow \\
& \langle\mathbf{p}|=\langle\mathbf{a}| *\langle\mathbf{b}|=\left\{p_{I}=a_{I} b_{I} \mid I=1, N\right\}:\langle\mathbf{p}| \in \mathbb{V}_{N}[\mathbb{N}]
\end{aligned}
$$

The row vector has been chosen for ease of writing, but everything could be described in a column vector space and the Dirac's ket notation: $|\mathbf{a}\rangle=\left(a_{1}, a_{2}, a_{3}, \ldots a_{N}\right)^{T}$, where the superscript $T$ means transposition of the row vector into a column one.

Such an inward product, which allows the natural vector spaces to behave like the natural number set, has been previously named as diagonal, Hadamard, or Schur product.

The inward product of two vectors behaves like the product of scalars. Therefore, the inward product is associative, commutative, and distributive concerning the 
vector sum. It can be extended without effort to matrix-vector spaces. Obviously enough, inward products can involve as many vectors as inward factors as needed. No other natural vector properties are needed for the definition of Fermat vectors.

\subsection{Complete sum of a vector}

The complete sum (of the elements) of a vector: $\langle\langle\mathbf{a} \mid\rangle$, can be defined as a linear operator acting on a vector yielding a scalar. That is:

$$
\forall\langle\mathbf{a}| \in \mathbb{V}_{N}[\mathbb{N}]:\langle\mathbf{a}|=\left\{a_{I} \mid I=1, N\right\}:\left\langle\langle\mathbf{a} \mid\rangle=\sum_{I=1}^{N} a_{I} \rightarrow\langle\langle\mathbf{a} \mid\rangle \in \mathbb{N},\right.
$$

and it is trivial to show that:

$$
\forall\{\langle\mathbf{a}|,\langle\mathbf{b}|\} \subset \mathbb{V}_{N}[\mathbb{N}] \rightarrow\langle\langle\mathbf{a}|+\langle\mathbf{b} \mid\rangle=\langle\langle\mathbf{a} \mid\rangle+\langle\langle\mathbf{b} \mid\rangle,
$$

and that:

$$
\forall\langle\mathbf{a}| \in \mathbb{V}_{N}[\mathbb{N}] \wedge \alpha \in \mathbb{N}:\langle\alpha\langle\mathbf{a} \mid\rangle=\alpha\langle\langle\mathbf{a} \mid\rangle
$$

showing that the complete sum operator is linear.

\subsection{Second-order scalar product}

The inward product of two vectors and the complete sum operator can be used together to redefine the scalar product of two vectors, which due to the possibility to describe higher-order products of this kind using the same operations, will be named as a second-order scalar product.

Using definitions (2) and (3) a second-order scalar product is immediately defined as:

$$
\forall\{\langle\mathbf{a}|,\langle\mathbf{b}|\} \subset \mathbb{V}_{N}[\mathbb{N}]:\left\langle\langle\mathbf{a}| *\langle\mathbf{b} \mid\rangle=\sum_{I=1}^{N} a_{I} b_{I} \in \mathbb{N} \rightarrow\langle\langle\mathbf{a}| *\langle\mathbf{b} \mid\rangle \equiv\langle\mathbf{a} \mid \mathbf{b}\rangle\right.
$$

\section{4 -th order scalar product}

One can now consider the inward product of $p$ vectors as another vector belonging to the same vector space, or:

$$
\begin{aligned}
& \left\{\left\langle\mathbf{a}_{I}|| I=1, p\right\} \subset \mathbb{V}_{N}[\mathbb{N}]: \underset{I=1}{*}\left\langle\mathbf{a}_{I}\right| \in \mathbb{V}_{N}[\mathbb{N}] \rightarrow\right. \\
& \left\langle\underset{I=1}{*}\left\langle\mathbf{a}_{I} \mid\right\rangle=\sum_{J=1}^{N}\left(\prod_{I=1}^{p} a_{I J}\right) \in \mathbb{N},\right.
\end{aligned}
$$

Therefore, the Eq. (7) defines a $p$-th order scalar product involving $p$ vectors. 


\section{5 p-th order power of a vector}

In the same manner, as defining higher-order inward products, one can use the repeated inward product of a vector, constructing in this way a $p$-th natural power of a vector:

$$
\forall\langle\mathbf{a}| \in \mathbb{V}_{N}[\mathbb{N}]:\left\langle\mathbf{a}^{[p]}|=\underset{I=1}{*} \underset{*}{*}| \in \mathbb{V}_{N}[\mathbb{N}] \rightarrow\left\langle\mathbf{a}^{[p]}\right|=\left\{a_{I}^{p} \mid I=1, N\right\}\right.
$$

\section{6 p-th order norm of a natural vector}

The previous Eqs. (3) and (8) permit to define the $p$-th order norm of a vector, $\mathrm{N}_{p}[\langle\mathbf{a}|]$, because using the previously defined operations one can easily write:

$$
\forall\langle\mathbf{a}| \in \mathbb{V}_{N}[\mathbb{N}]: \mathrm{N}_{p}[\langle\mathbf{a}|]=\left\langle\left\langle\mathbf{a}^{[p]} \mid\right\rangle=\sum_{I=1}^{N} a_{I}^{p} \in \mathbb{N} .\right.
$$

Natural spaces, from the point of view of the existence of Eq. (9), can be also associated with Banach spaces, where a set of $p$-th order norms are well-defined.

\section{Minkowski natural spaces and $p$-th order vector norms.}

From the Euclidean structure of natural vector spaces, as described in the previous paragraph, an $(N+1)$-dimensional Minkowski natural space $\mathbb{M}_{(N+1)}(\mathbb{N})$ can be easily constructed. For more information, readers are referred to reference [12].

Now, a Minkowski space is an $(N+1)$-dimensional natural space, where a metric vector $\langle\mathbf{m}|$, associated with the space norms, can be constructed using the structure:

$$
\langle\mathbf{m}|=(\langle\mathbf{1}| ;-1),
$$

where the vector $\langle\mathbf{1}|=(1,1, \ldots, 1)$ is featured as the $N$-dimensional unity vector.

In this manner, having defined the metric vector (10), the $p$-th order norm $\mathbf{M}_{p}(\langle\mathbf{a}|)$ of a vector in a Minkowski natural space can be easily redefined. This might be performed using the complete sum of an inward product as in the Eq. (9), but including the metric vector (10) in the definition:

$$
\forall\langle\mathbf{a}| \in \mathbb{M}_{(N+1)}(\mathbb{N}): \mathbf{M}_{p}(\langle\mathbf{a}|)=\left\langle\langle\mathbf{m}| *\left\langle\mathbf{a}^{[p]} \mid\right\rangle=\sum_{I=1}^{N} a_{I}^{p}-a_{N+1}^{p}\right.
$$




\section{Fermat vectors and Fermat's last theorem.}

A vector $\langle\mathbf{f}| \in \mathbb{M}_{(N+1)}(\mathbb{N})$ belonging to a Minkowski natural space can be called a Fermat vector of order $p$ (or of $p$-th order) whenever the following equality:

$$
\mathrm{M}_{p}(\langle\mathbf{f}|)=0
$$

holds.

Using the definition of the Fermat vectors via Eqs. (11) and (12), the so-called Fermat's last theorem implies the following equation:

$$
\exists\langle\mathbf{f}| \in \mathbb{M}_{(2+1)}(\mathbb{N}): M_{p=2}(\langle\mathbf{f}|)=0 \wedge \forall\langle\mathbf{v}| \in \mathbb{M}_{(2+1)}(\mathbb{N}): M_{p>2}(\langle\mathbf{v}|) \neq 0,
$$

is accomplished.

\section{Details of the computational search of Fermat vectors.}

Calculations in the search of Fermat vectors have been performed within a discrete set of $2^{M}$ natural numbers, associated with the set $\mathbb{S}_{M}=\left\{0,1,2, \ldots,\left(2^{M}-1\right)\right\} \subset \mathbb{N}$, related to the decimal representation of the bit strings of an $M$-dimensional Boolean Hypercube, see for example reference [16].

One can refer to the number of Fermat vectors found in a natural number batch $\mathbb{S}_{M}$, using a Minkowski vector space dimension $(N+1)$ and an order $p$, with the symbol: $\#(M, N, p)$. Then it is obtained, that: $M_{0}<M_{1} \Rightarrow \#\left(M_{0} ; N, p\right) \leq \#\left(M_{1} ; N, p\right)$

However, obviously enough the computational time strongly increases as the value of the Hypercube dimension increases. In the computations presented in reference [6] and here, the maximal value of the hypercube dimension has been set to $M=15$.

To ease the calculation involving large powers of the elements of the natural set $\mathbb{S}_{M}$, a vector containing the $p$-th power of this natural set:

$$
\mathbb{S}_{M}^{[p]}=\left\{0,1,2^{p}, 3^{p}, \ldots,\left(2^{M}-1\right)^{p}\right\} \subset \mathbb{N}^{[p]} \subset \mathbb{N}
$$

is previously computed and by $N^{[p]}$ one can suppose that is described the set of all natural numbers to the $p$-th power. Then, the search of Fermat vectors will take place in a subset of the natural vector space, whose vectors are made with the elements of the powers of natural numbers, as defined by the Eq. (14). That is, with the vectors:

$$
\forall\left\langle\mathbf{v}^{[p]}\right| \in \mathbb{U}_{(N+1)}\left(\mathbb{S}_{M}^{[p]}\right) \subset \mathbb{M}_{(N+1)}(\mathbb{N}) .
$$

Still, when large Minkowski space dimensions are tested, the combinatorial explosion of all the possible created vectors might skyrocket the computing time to unreachable values, of course considering the authors' limited number of available 
computers. The evolution of computing structure perhaps will permit to obtain extended information on this subject soon.

The vectors $\left\langle\mathbf{v}^{[p]}\right|$ described through the Eqs. (14) and (15), tested for being Fermat's vectors, are constructed without containing nor the zero nor the unit values as elements.

\section{Fermat vectors of order 2.}

The classical Fermat theorem, proved by Wiles [13], amounts the same as to consider that the following equation holds:

$$
\exists\langle\mathbf{f}| \in \mathbb{M}_{(2+1)}(\mathbb{N}): M_{2}(\langle\mathbf{f}|)=0 \wedge \forall\langle\mathbf{v}| \in \mathbb{M}_{(2+1)}(\mathbb{N}): M_{p \geq 3}(\langle\mathbf{v}|) \neq 0,
$$

which constitutes another way to express the Eq. (13).

To test the second-order first part of the Eq. (16), a large number of computations with Minkowski natural spaces of diverse dimensions have been performed.

Computational results have shown that the next expression:

$$
\forall N \geq 2: \exists\langle\mathbf{f}| \in \mathbb{M}_{(N+1)}(\mathbb{N}): \mathrm{M}_{2}(\langle\mathbf{f}|)=0,
$$

stands for a wide range of dimensions. The largest dimension tested in the Eq. (17) has been $N=601$. Computational results coherent with the Eq. (17) suggest it seems possible one can conjecture that the above equation holds for indefinite natural space dimension values.

This is the same to conjecture that: second-order Fermat vectors exist for any natural Minkowski space dimension.

One must, in this context of Fermat vectors of order 2, refer to some Leech and Lorentzian lattices, which correspond to 24-dimensional Fermat vectors of order 2 in our notation [14].

Nevertheless, second-order Fermat $N$-dimensional vectors represent points, possessing natural coordinates, on the surface of an $N$-dimensional sphere with a radius set at the Minkowski coordinate in the $N+1$ element.

\section{Fermat vectors of order 3.}

Recent exhaustive supercomputations [6] in Minkowski's natural spaces of dimension $(3+1)$ have shown a behavior similar to the vectors of the lesser dimension $(2+1)$. That is, empirical computational evidence allows to conjecture that the equation:

$$
\exists\langle\mathbf{f}| \in \mathbb{M}_{(3+1)}(\mathbb{N}): M_{p \leq 3}(\langle\mathbf{f}|)=0 \wedge \forall\langle\mathbf{v}| \in \mathbb{M}_{(3+1)}(\mathbb{N}): M_{p>3}(\langle\mathbf{v}|) \neq 0
$$

holds. Indicating that an extended Fermat's theorem can be postulated on dimension $(3+1)$ in the same manner as on dimension $(2+1)$. That might be stated as the fact that the Eq. (18) is the higher-dimensional extension of the Eq. (16). 
However, one thing is to obtain consistent computational results, and another the demonstration of such an extended Fermat's theorem conjecture.

Also, in the same way, as found in the second-order norms, as shown in the Eq. (17), there are Fermat vectors in higher Minkowski natural space dimensions than $N=3$ that accept null third-order norms. That is, one can write another statement, as an equivalent extended conjecture, described by the Eq. (17):

$$
\forall N \geq 3: \exists\langle\mathbf{f}| \in \mathbb{M}_{(N+1)}(\mathbb{N}): \mathrm{M}_{3}(\langle\mathbf{f}|)=0,
$$

the largest dimension tested on the Eq. (19) has been in this case: $N=151$.

In the light of the computational experience, one can conjecture that Minkowski natural spaces of dimensions $(2+1)$ and $(3+1)$ behave similarly concerning the existence and absence of Fermat vectors of orders 2 and 3.

\section{Fermat vectors of order $p>3$.}

In some cases, the search for Fermat vectors of higher orders has been exhaustive as in the lower order cases, commented in the previous paragraphs. In other issues, the search has not been so extensively performed because of the combinatorial difficulty that the calculations present. Some aspects of the computation of Fermat vectors, which also can be applied to the previously discussed dimensions and orders, will be given next.

\subsection{Computational details}

However, we can say that besides the supercomputing search, performed according to the reference [6], some tests have been achieved in i7 and i9 desktop computers, through Python code.

Such a code does not use the whole possible set of natural vectors, as defined in the Eqs. (14) and (15), but a randomly chosen subset amounting to a selected percent of the total number of candidate vectors as defined in the Eq. (15) to be tested. Such a procedure permits obtaining Fermat vectors within a reasonable computational time. Results from such random calculations have produced coincident matches with the exhaustive tests performed in a supercomputer environment. Such coincidences can be seen as a way to empirically validate the proposed conjectures.

\subsubsection{On the infinite cardinality of Fermat's vectors}

Note that, when a Fermat vector $\langle\mathbf{f}|$ of $p$-th order: $\left\langle\mathbf{f}^{[p]}\right|$ is found, this just means that there exist an infinite number of Fermat vectors, as all the homothetic vectors obtained as:

$$
\mathbf{M}_{p}[\langle\mathbf{f}|]=0 \wedge \forall \lambda \in \mathbb{N}:\left\langle\mathbf{f}_{\lambda}^{[p]}\right|=\lambda^{p}\left\langle\mathbf{f}^{[p]}\right| \rightarrow \mathbf{M}_{p}[\lambda\langle\mathbf{f}|]=0
$$


also possess the corresponding $p$-th order norm null.

\subsection{The case of dimension $(4+1)$ : meta-Fermat vectors}

It could have been interesting to find out a comparable behavior for higher dimensional Minkowski natural spaces, similar to the results obtained in the previously described paragraphs, concerning lesser dimensions.

To have some hint about the possibility to extend the Fermat theorem to higher dimensions, several computational tests have been performed within Minkowski spaces of dimension $(4+1)$. The obtained computational results permit that the following general statement:

$$
\exists\langle\mathbf{f}| \in \mathbb{M}_{(4+1)}(\mathbb{N}): M_{p \leq 5}(\langle\mathbf{f}|)=0 \wedge \forall\langle\mathbf{v}| \in \mathbb{M}_{(4+1)}(\mathbb{N}): M_{p>5}(\langle\mathbf{v}|) \neq 0,
$$

might be conjectured. The Eq. (21) perhaps shows that in Minkowski natural spaces of greater dimension there could appear anomalous meta-extensions of Fermat's theorem, like the one found in $(4+1)$ dimensional Minkowski natural space, where Fermat vectors of dimension $(4+1)$ and order 5 have been found.

\subsection{The higher dimensions case.}

Computations with a large number of vectors and diverse Minkowski natural space dimensions show that it seems the meta-Fermat vector extension of order 5 in $(4+1)$ dimension, does not easily appear in the tested higher dimensions $([N>4]+1)$. On the contrary, as the Minkowski natural space dimension grows larger, it is more difficult to find higher-order Fermat vectors.

In the light of the large set of numerical tests performed, one can conjecture the following statement, though:

$$
\forall N \geq 5: \exists\langle\mathbf{f}| \in \mathbb{M}_{(N+1)}(\mathbb{N}): \mathbf{M}_{p \leq 5}(\langle\mathbf{f}|)=0 .
$$

Results with larger order Minkowski norms have yielded no Fermat vectors wearing a zero norm. However, such a result does not signify that they do not exist. Simply under the computational constraints used no vector of this kind was found.

It looks as the zero norms of order 5 constitute some limit, which the performed numerical computational analysis has been unable to trespass.

As a consequence, it is not advisable to transform this last finding, contained in the Eq. (22), into a conjecture, as nothing opposes obtaining, ahead in time, zero norms within larger orders and bigger dimension spaces.

It is a matter of computer power and calculation costs to find out.

Alternatively, one can rely on the plausible development of a theoretical structure, able to explain the detailed nuances about the existence of the Fermat vectors in complicated vector landscapes, similar to the one which was performed by Wiles [13] within the order 2 and the $(2+1)$ dimensional case. 


\subsection{Resumé}

Fermat's last theorem originally set up in spaces of $(2+1)$ dimensions seem extensible to Minkowski's natural spaces of dimension $(3+1)$. Also, in dimension $(4+1)$, there appears that Fermat vectors with zero norms up to order 5 might be found. Therefore, a conjecture extending a Fermat theorem up to this anomalous order number seems to be conceivable.

Higher dimensions provide Fermat vectors of lower order in abundance but, for instance, dimension $(5+1)$ provides a scarce amount of Fermat vectors of order 5. Such scarcity makes it difficult to extend the Fermat theorem up to this dimension, although it seems plausible that it can be so.

Higher dimensions $([N>5]+1)$ produce Fermat vectors, but of orders $\leq 5$. A fact which thwarts the possibility to extend a Fermat theorem conjecture upwards from Minkowski's natural spaces of dimension $(5+1)$. Unless high-speed computations, better than the ones used here can be tested in the future.

\section{Fermat hypersurfaces and the scarcity of Fermat vectors}

The reason for the difficulty of finding Fermat vectors of higher orders and dimensions is not at all easy to explain, though. The culprit of this complexity can be associated with many factors, which perhaps will appear more clearly when extensive computations could be developed, dedicated to shedding light on the search of Fermat vectors of higher dimensions and orders. Whenever one can overcome the combinatorial explosion of the generated natural vectors within larger dimensions and the corresponding bigger orders.

Perhaps, to understand a little bit better the problem we are facing, one might use the fact that vector powers in Minkowski natural spaces represent points, bearing coordinates made solely of natural numbers, but contained within a high dimensional surface, mostly defined over the rational (or real) field.

A Fermat hypersurface might be described within a Minkowski semispace as:

$$
\exists p \in \mathbb{N} \wedge r \in \mathbb{Q}^{+} \wedge \exists\langle\mathbf{x}| \in \mathbb{V}_{N}\left(\mathbb{Q}^{+}\right): r^{p}=\sum_{I=1}^{N} x_{I}^{p} \equiv\left\langle\left\langle\left\langle\mathbf{x}^{[p]}\left|;-r^{p}\right|\right\rangle=0,\right.\right.
$$

with the parameter $r$ taken as a constant. It must be noted that, when the order is $p=2$, the hypersurface of the Eq. (23) represents an $N$-dimensional sphere of radius $r$.

The probability that a point, lying into a higher dimensional, higher-order rational (or real) hypersurface, could coincide with coordinates made by powers of natural numbers, seems that decreases significantly by augmenting the dimension and the order of the hypersurface described in the Eq. (23).

That is, associating the right side of the Eq. (23) with a function: 


$$
F_{p}\left[\langle\mathbf{x}|| r]=\sum_{I=1}^{N} x_{I}^{p}-r^{p} \rightarrow F_{p}[\langle\mathbf{x}|| r]=0,\right.
$$

for a constant parameter $r$, one can say there is an infinite number of vectors $\langle\mathbf{x}| \in \mathbb{V}_{N}\left(\mathbb{Q}^{+}\right)$fulfilling the Eq. (24).

Now, a Fermat vector, like the one defined in the Eq. (12), will fit into such a function as in the Eq. (24), taking into account the use of a constant natural parameter $r$, in the same way as the vector defined with:

$$
\exists r, p \in \mathbb{N} \wedge \exists\langle\mathbf{f}| \in \mathbb{V}_{N}(\mathbb{N}): F_{p}[\langle\mathbf{f}|| r]=0 .
$$

This fact plausibly produces a dramatic scarcity of Fermat vectors when incrementing the space dimension and the order of the associated norms. An empirical fact that has been computationally observed.

Performing computations in search of Fermat vectors, when the parameter $r$, the power $p$, and a vector $\langle\mathbf{f}|$ are found, fulfilling the Eq. (25), then one can say such occurrence corresponds to find a unique natural point fulfilling the function (24).

In other words, as far as we know, every element of a large number of obtained Fermat vectors corresponds to a unique natural position on some Fermat hypersurface of order $p$ and radius $r$, defined as shown in Eqs. (23) or (24).

This can explain perhaps the observed computational fact that Fermat vectors, in case they exist, become more and more scarce when Minkowski space dimension and norm order become larger than 5 .

\subsection{Some test computations describing Fermat vectors associated with the same hypersurface.}

Some extra computation search of Fermat vectors has been performed using the aleatory algorithm described before in Sect. 8.1, to illustrate the nature of Fermat vectors as natural points in a Fermat hypersurface.

Interesting results corresponding to Fermat vectors possessing the same parameter $r$ can be found as explained below in various computation batches, attached to the same space and power, indicating the nature of Fermat vectors as different unique natural points belonging to the same Fermat hypersurface attached to the constant parameter $r$.

For batches associated with \#(7,4,3) several vector triples having the same parameter $r$ have been found. For batches like \#(7,3,2) even some quadruple Fermat vectors possessing the same parameter $r$ had resulted from the computation.

It is interesting to note that $\#(7,10, p)[p=6,7,8]$ yield, after several days of computation, no Fermat vectors. However, using $[p=3,4,5]$, apart from the existence of Fermat vectors, at every value of $p$, many pairs of Fermat vectors with the same parameter $r$ did appear.

A test of large dimensions has been also performed in the form $\#(7,25, p)[p=4,5]$, with the result of obtaining several vectors containing scarce Fermat vector pairs, with the same parameter $r$, when the lower power $[p=4]$ was 
observed, but no Fermat vectors at all were generated with the higher power value $[p=5]$.

When both dimension and power raise, the number of possible Fermat vectors increases to an exceptionally large amount, even if the generating Mersenne power is not too big.

This behavior of Fermat vectors can be used empirically to explain the scarcity of such vectors as the parameters $\#(M, N, p)$ become larger.

\section{Conclusions}

As a result of exhaustive computations to find out $p$-th order Fermat vectors in $(N+1)$ dimensional natural Minkowski spaces, one can empirically extend Fermat's last theorem beyond the $(2+1)$ dimensional spaces.

Certainly, such findings are empirical, therefore the obtained results shall be formulated in the form of a conjecture.

One can describe a plausible $(N+1)$ dimensional Fermat conjecture, in the form provided by Eqs. (16), (17), (18), (19), (21), and (22).

That there cannot be used a unique expression is due to the anomalous behavior encountered at the dimension $(4+1)$.

Something similar could be found in higher dimensions. However, for the moment, the computationally explosive nature of the combinatorial problem, associated with the search for Fermat vectors of higher dimensions, has not provided another comparable result.

Acknowledgements One of us (R. C.-D.) wishes to express his gratitude to Prof. Carlos Perelman for his helpful comments about this manuscript.

Funding Open Access funding provided thanks to the CRUE-CSIC agreement with Springer Nature.

\section{Declaration}

Conflict of interest The authors state that there is no conflict of interest related to this work.

Open Access This article is licensed under a Creative Commons Attribution 4.0 International License, which permits use, sharing, adaptation, distribution and reproduction in any medium or format, as long as you give appropriate credit to the original author(s) and the source, provide a link to the Creative Commons licence, and indicate if changes were made. The images or other third party material in this article are included in the article's Creative Commons licence, unless indicated otherwise in a credit line to the material. If material is not included in the article's Creative Commons licence and your intended use is not permitted by statutory regulation or exceeds the permitted use, you will need to obtain permission directly from the copyright holder. To view a copy of this licence, visit http://creativecommons.org/licen ses/by/4.0/. 


\section{References}

1. R. Carbó-Dorca, Towards a universal quantum QSPR operator. Int. J. Quant. Chem. 118, 1-17 (2018)

2. R. Carbó-Dorca, Transformation of boolean hypercube vertices into unit interval elements: QSPR workout consequences. J. Math. Chem. 57, 694-696 (2019)

3. R. Carbó-Dorca, Universal transformation and non-linear connection between experimental and calculated property vectors in QSPR. J. Math. Chem. 57, 1075-1087 (2019)

4. R. Carbó-Dorca, Natural vector spaces, (inward power and Minkowski norm of a natural vector, natural boolean hypercubes) and a Fermat's last theorem conjecture. J. Math. Chem. 55, 914-940 (2017)

5. R. Carbó-Dorca, C. Muñoz-Caro, A. Niño, S. Reyes, Refinement of a generalized Fermat's last theorem conjecture in natural vector spaces. J. Math. Chem. 55, 1869-1877 (2017)

6. A. Niño, S. Reyes, R. Carbó-Dorca, An HPC hybrid parallel approach to the experimental analysis of Fermat's theorem extension to arbitrary dimensions on heterogeneous computer systems. J. Supercomput. (2021). https://doi.org/10.1007/s11227-021-03727-2

7. K. Sen, R. Carbó-Dorca, Inward matrix products, generalized density functions, and Rayleigh Schrödinger perturbation theory. J. Mol. Struct. Theochem 501, 173-176 (2000)

8. R. Carbó-Dorca, Inward matrix products: extensions and applications to quantum mechanical foundations of QSAR. J. Mol. Struct. Teochem 537, 41-54 (2001)

9. R. Carbó-Dorca, Inward matrix product algebra and calculus as tools to construct space-time frames of arbitrary dimensions. J. Math. Chem. 30, 227-245 (2001)

10. R. Carbó-Dorca, E. Besalú, Shells, point cloud huts, generalized scalar products, cosines and similarity tensor representations in vector semispaces. J. Math. Chem. 50, 210-219 (2012)

11. R. Carbó-Dorca, Boolean Hypercubes and the structure of vector spaces. J. Math. Sci. Mod. (JMSM) 1, 1-14 (2018)

12. R. Carbó-Dorca, Generalized scalar products in Minkowski metric spaces. J. Math. Chem. 59, 1029-1045 (2021)

13. A. Wiles, Modular elliptic-curves and Fermat's last theorem. Ann. Math. 141, 443-551 (1995)

14. See for example the website: https://en.wikipedia.org/wiki/Leech_lattice\#Using_the_Lorentzian_ lattice_II 25,1

15. R. Carbó-Dorca, T. Chakraborty, Extended Minkowski spaces, Zero norms, and Minkowski Surfaces. SQMM-CERT Technical Report TC-2021-5.

16. R. Carbó-Dorca, Boolean Hypercubes, Mersenne numbers, and the Collatz conjecture. J. Math. Sci Modelling. 3, 120-129 (2020)

Publisher's Note Springer Nature remains neutral with regard to jurisdictional claims in published maps and institutional affiliations. 